\title{
Overview on elliptic multiple zeta values
}

Nils Matthes

\begin{abstract}
We give an overview of some work on elliptic multiple zeta values. First defined by Enriquez as the coefficients of the elliptic KZB associator, elliptic multiple zeta values are also special values of multiple elliptic polylogarithms in the sense of Brown and Levin. Common to both approaches to elliptic multiple zeta values is their representation as iterated integrals on a once-punctured elliptic curve. Having compared the two approaches, we survey various recent results about the algebraic structure of elliptic multiple zeta values, as well as indicating their relation to iterated integrals of Eisenstein series, and to a special algebra of derivations.
\end{abstract}

\section{Introduction}

The purpose of this article is twofold. The first goal is to unify two different approaches to elliptic multiple zeta values in the literature: the approach of Enriquez, using elliptic associators [21, 22] on one hand, and the approach of Brown and Levin, using multiple elliptic polylogarithms [15] on the other. Comparing the two approaches also highlights the analogy between elliptic multiple zeta values and multiple zeta values. The second goal is to collect various results on the structure of

Nils Matthes

Fachbereich Mathematik (AZ)

Universität Hamburg

Bundesstrasse 55, D-20146 Hamburg

e-mail: nils.matthes@uni-hamburg.de

Current address:

Mathematical Institute, University of Oxford

Andrew Wiles Building, Radcliffe Observatory Quarter

Woodstock Road, Oxford OX2 6GG, United Kingdom

e-mail: nils.matthes@maths.ox.ac.uk 
the algebra of A-elliptic multiple zeta values, which appeared in work of the author [36], as well as in joint work $[5,6]{ }^{1}$

\subsection{Multiple zeta values}

Multiple zeta values are defined by nested sums

$$
\zeta\left(n_{1}, \ldots, n_{r}\right)=\sum_{0<k_{1}<\ldots<k_{r}} \frac{1}{k_{1}^{n_{1}} \ldots k_{r}^{n_{r}}} \in \mathbb{R},
$$

for integers $n_{1}, \ldots, n_{r} \geq 1$, with $n_{r} \geq 2$. The weight of $\zeta\left(n_{1}, \ldots, n_{r}\right)$ is $n_{1}+\ldots+n_{r}$, and its depth is $r$. For $r=1$, they are the values of the Riemann zeta function at positive integers. Multiple zeta values appear in mathematics and physics in many different contexts, for example in the context of periods and motives $[9,19,26]$. Also, multiple zeta values arise naturally in the study of the Knizhnik-Zamolodchikov (KZ) equation from quantum field theory [20, 29], which in turn is important in both knot theory [30], as well as quantum groups [20] and associators [23]. On the side of mathematical physics, multiple zeta values occur in the computation of Feynman integrals in renormalizable quantum field theories [4], and in the computation of superstring amplitudes at tree-level [7, 8, 40].

In all of these contexts, a central problem is to find a complete description of all $\mathbb{Q}$-linear relations between multiple zeta values. The $\mathbb{Q}$-vector subspace $\mathscr{Z} \subset$ $\mathbb{R}$, spanned by the multiple zeta values is a $\mathbb{Q}$-subalgebra of $\mathbb{R}$. Conjecturally, the weight is a grading on $\mathscr{Z}$, while the depth defines an ascending filtration on $\mathscr{Z}$. Set

$$
\mathscr{D}_{d} \mathscr{Z}_{N}=\left\langle\zeta\left(n_{1}, \ldots, n_{r}\right) \in \mathscr{Z} \mid n_{1}+\ldots+n_{r}=N, r \leq d\right\rangle_{\mathbb{Q}} .
$$

The Broadhurst-Kreimer conjecture [4] gives a formula for the dimensions of the associated graded $D_{N, d}=\operatorname{dim}_{\mathbb{Q}} \operatorname{gr}_{d}^{\mathscr{D}}\left(\mathscr{Z}_{N}\right):=\mathscr{D}_{d}\left(\mathscr{Z}_{N}\right) / \mathscr{D}_{d-1}\left(\mathscr{Z}_{N}\right)$, and suggests a relation between multiple zeta values and modular forms for $\mathrm{SL}_{2}(\mathbb{Z})[11,12,24$, 25]. Work of Zagier [45] and Goncharov [25] implies that the Broadhurst-Kreimer formula gives an upper bound in depths $d \leq 3$, for arbitrary $N$.

\subsection{The algebra of A-elliptic multiple zeta values}

In [22], Enriquez introduced an elliptic analogue of multiple zeta values, which has found recent applications in superstring theory [5]. Elliptic multiple zeta values are defined by integrating the elliptic Knizhnik-Zamolodchikov-Bernard (KZB) connection [16] along paths on a once-punctured elliptic curve $E_{\tau}^{\times}=\mathbb{C} /(\mathbb{Z}+\mathbb{Z} \tau) \backslash\{0\}$ with $\tau \in \mathbb{H}$. There are essentially two choices of paths on $E_{\tau}^{\times}$, corresponding to the

\footnotetext{
${ }^{1}$ See also Section 1.4 .
} 
two natural homology cycles $\alpha$ and $\beta$ on the elliptic curve, giving rise to two algebras of elliptic multiple zeta values, namely A- and B-elliptic multiple zeta values. The two types of elliptic multiple zeta values are related to each other by a modular transformation formula [22] and, for simplicity, we will only consider A-elliptic multiple zeta values.

The $\mathbb{Q}$-vector space spanned by A-elliptic multiple zeta values is a $\mathbb{Q}$-subalgebra $\mathscr{E} \mathscr{Z}^{\mathrm{A}}$ of the $\mathbb{C}$-algebra $\mathscr{O}(\mathbb{H})$ of holomorphic functions on the upper half-plane $\mathbb{H}$. The analogous notions of weight and depth for multiple zeta values are the weight and the length for A-elliptic multiple zeta values. Denote by $\mathscr{E} \mathscr{Z}_{N}^{\mathrm{A}} \subset \mathscr{E} \mathscr{Z}^{\mathrm{A}}$ the subspace spanned by A-elliptic multiple zeta values of weight $N$. The analogue of a conjecture of Zagier's for multiple zeta values is:

Conjecture 1. The weight defines a grading on $\mathscr{E} \mathscr{Z}^{\mathrm{A}}$, i.e.

$$
\mathscr{E} \mathscr{Z}^{\mathrm{A}}=\bigoplus_{N \geq 0} \mathscr{E} \mathscr{Z}_{N}^{\mathrm{A}}
$$

Similar to the case of multiple zeta values, the length defines an ascending filtration $\mathscr{L}_{\bullet}\left(\mathscr{E} \mathscr{Z}_{N}^{\mathrm{A}}\right)$, and we denote by $\operatorname{gr}_{\bullet}^{\mathscr{L}}\left(\mathscr{E} \mathscr{Z}_{N}^{\mathrm{A}}\right)$ the associated graded. In light of the Broadhurst-Kreimer conjecture for multiple zeta values, it is natural to pose:

Problem 1. Compute the dimension

$$
D_{N, l}^{\mathrm{ell}}=\operatorname{dim}_{\mathbb{Q}} \operatorname{gr}_{l}^{\mathscr{L}}\left(\mathscr{E} \mathscr{Z}_{N}^{\mathrm{A}}\right)
$$

of the space of A-elliptic multiple zeta values of weight $N$ and length $l$, for all $N, l \geq 0$.

A first step towards a solution to this problem is taken in [36].

Theorem 1. We have

$$
D_{N, 0}^{\text {ell }}=\delta_{N, 0}, \quad D_{N, 1}^{\text {ell }}= \begin{cases}1 & N \geq 2 \text { even } \\ 0 & \text { else },\end{cases}
$$

and

$$
D_{N, 2}^{\text {ell }}= \begin{cases}0 & N \text { even } \\ \left\lfloor\frac{N}{3}\right\rfloor+1 & N \text { odd } .\end{cases}
$$

Also, we have the weight grading in lengths 0,1 and 2, i.e., for $l=0,1,2$, we have

$$
\mathscr{L}_{l}\left(\mathscr{E} \mathscr{Z}^{\mathrm{A}}\right)=\bigoplus_{N \geq 0} \mathscr{L}_{l}\left(\mathscr{E} \mathscr{Z}_{N}^{\mathrm{A}}\right)
$$

The proof uses a linear independence result for a certain family of elliptic multiple zeta values, as well some basic invariant theory. For more details, see Section 4.3, and also [6], in particular Section 4.5 for related work in higher lengths. 


\subsection{Structure of the article}

In Section 2, we give a very brief overview on multiple zeta values, multiple polylogarithms and the Drinfeld associator. This section is primarily intended to make the analogy with elliptic multiple zeta values transparent. In Section 3, we introduce elliptic multiple zeta values, comparing the works of Enriquez and of Brown and Levin. The main ingredient in both approaches, namely the universal elliptic KZB equation [16, 32], is introduced in Section 3.1.

In Section 4, we begin the study of A-elliptic multiple zeta values. All of the results of this section can be found in $[5,6,36]$, and are primarily concerned with explicit algebraic relations between A-elliptic multiple zeta values. Finally, in Section 5, we draw the connection between A-elliptic multiple zeta values, iterated integrals of Eisenstein series, and a special algebra of derivations $\mathfrak{u}^{\text {geom }}$ on a free Lie algebra on two generators [13, 16, 32, 37].

\subsection{Note added in print}

This article was written in 2015 and therefore does not reflect more recent progress on the subject of elliptic multiple zeta values. See in particular [33] for a study of double shuffle relations in the context of elliptic multiple zeta values and the author's $\mathrm{PhD}$ thesis [35] for a more detailed discussion of elliptic multiple zeta values.

\section{Multiple polylogarithms and the Drinfeld associator}

Multiple zeta values can be defined either as special values of multiple polylogarithms, or equivalently as the coefficients of Drinfeld's associator. A common theme of the two definitions is the theory of iterated integrals on $\mathbb{P}^{1} \backslash\{0,1, \infty\}$. Of particular importance is the concept of homotopy invariance of iterated integrals, because of the connection to the unipotent fundamental group of $\mathbb{P}^{1} \backslash\{0,1, \infty\}$.

\subsection{Iterated integrals}

We begin with a brief reminder on iterated integrals. More background can be found for example in $[17,27]$.

Let $M$ be a smooth manifold over $k$, where $k$ denotes either the field of real or complex numbers, $\gamma:[0,1] \rightarrow M$ a piecewise smooth path, and $\left(\omega_{1}, \ldots, \omega_{r}\right)$ an $\mathrm{r}-$ tuple of smooth differential one-forms on $M$. Write $f_{i}(t) d t$ for the pullback $\gamma^{*}\left(\omega_{i}\right)$ of $\omega_{i}$ along $\gamma$. Then we define 


$$
\int_{\gamma} \omega_{1} \ldots \omega_{r}=\int_{0}^{1} f_{1}\left(t_{1}\right) \int_{0}^{t_{1}} f_{2}\left(t_{2}\right) \ldots \int_{0}^{t_{r-1}} f_{r}\left(t_{r}\right) d t_{r} \ldots d t_{2} d t_{1}
$$

and call this integral an iterated integral. If $r=0$, then we define $\int_{\gamma} \equiv 1$. Many properties of iterated integrals are known, for example the shuffle product formula [39]

$$
\int_{\gamma} \omega_{1} \ldots \omega_{r} \int_{\gamma} \omega_{r+1} \ldots \omega_{r+s}=\sum_{\sigma \in \Sigma_{r, s}} \int_{\gamma} \omega_{\sigma^{-1}(1)} \ldots \omega_{\sigma^{-1}(r+s)},
$$

where $\Sigma_{r, s}$ denotes the set of all $(r, s)$-shuffles, i.e. $\Sigma_{r, s}$ consists of all permutations on the set $\{1, \ldots r+s\}$, which preserve the order of the first $r$ elements and the order of the last $s$ elements.

Now let $P M$ denote the set of all piecewise smooth paths on $M$. Fixing an $r$-tuple $\left(\omega_{1}, \ldots, \omega_{r}\right)$ of differential forms as above, we obtain a function

$$
\begin{aligned}
\int \omega_{1} \ldots \omega_{r}: P M & \rightarrow k \\
\gamma & \mapsto \int_{\gamma} \omega_{1} \ldots \omega_{r} .
\end{aligned}
$$

We call $\int \omega_{1} \ldots \omega_{r}$ a homotopy invariant iterated integral, if (10) depends only on the homotopy class of $\gamma$, i.e., if for every pair $\gamma_{0}, \gamma_{1} \in P M$ of paths, which are homotopic relative to their extremal points, we have $\int_{\gamma_{0}} \omega_{1} \ldots \omega_{r}=\int_{\gamma_{1}} \omega_{1} \ldots \omega_{r}$. The importance of homotopy invariant iterated integrals can be seen from Chen's $\pi_{1}$ de Rham theorem: the affine ring of functions on the unipotent completion of the fundamental group of a smooth manifold $M$ is given by the ring of all homotopy invariant iterated integrals on $M[17,27]$.

Finally, the following shorthand will come in useful later on. Denote by $\mathbb{C}\langle\langle x, y\rangle\rangle$ the $\mathbb{C}$-algebra of formal power series in non-commuting variables $x, y$, equipped with the concatenation product and let $I \subset \mathbb{C}\langle\langle x, y\rangle\rangle$ be the augmentation ideal, i.e. the two-sided ideal generated by $x$ and $y$. For a differential one-form $\omega$ with values in $I$, we set

$$
\exp \left[\int_{\gamma} \omega\right]=1+\sum_{k \geq 1} \int_{\gamma} \omega^{k} \in \mathbb{C}\langle\langle x, y\rangle\rangle
$$

where $\omega^{k}:=\underbrace{\omega \ldots \omega}_{k-\text { times }}$.

\subsection{Multiple polylogarithms}

From now on, we work over the field $\mathbb{C}$ of complex numbers. 


\subsubsection{Sum representation}

For integers $n_{1}, \ldots, n_{r} \geq 1$, define the multiple polylogarithm to be

$$
\operatorname{Li}_{n_{1}, \ldots, n_{r}}(z)=\sum_{0<k_{1}<\ldots<k_{r}} \frac{z^{k_{r}}}{k_{1}^{n_{1}} \ldots k_{r}^{n_{r}}} .
$$

The sum converges absolutely and locally uniformly for all $z \in \mathbb{C}$ with $|z|<1$, thus defines an analytic function on the open unit disk. Moreover, if $n_{r} \geq 2$, then (12) also converges for $z=1$, and in that case, $\operatorname{Li}_{n_{1}, \ldots, n_{r}}(1)$ is equal to the multiple zeta value $\zeta\left(n_{1}, \ldots, n_{r}\right)(1)$.

\subsubsection{Integral representation}

It follows directly from (12) that multiple polylogarithms satisfy the differential equation

$$
\frac{\partial}{\partial z} \operatorname{Li}_{n_{1}, \ldots, n_{r}}(z)= \begin{cases}\frac{1}{z} \mathrm{Li}_{n_{1}, \ldots, n_{r}-1}(z) & n_{r} \geq 2 \\ \frac{1}{1-z} \mathrm{Li}_{n_{1}, \ldots, n_{r-1}}(z) & n_{r}=1\end{cases}
$$

As a consequence multiple polylogarithms can be represented by iterated integrals. Consider the holomorphic differential one-forms $\omega_{0}=\frac{d z}{z}$ and $\omega_{1}=\frac{d z}{1-z}$ on $\mathbb{P}^{1} \backslash$ $\{0,1, \infty\}$. Then for $|z|<1$, one has

$$
\operatorname{Li}_{n_{1}, \ldots, n_{r}}(z)=\int_{0}^{z} \omega_{0}^{n_{r}-1} \omega_{1} \ldots \omega_{0}^{n_{1}-1} \omega_{1},
$$

where the path of integration is the straight line path from 0 to $z$. Note that although $\omega_{0}$ has a pole at 0 , the iterated integral is still well-defined, since the integration starts with $\omega_{1}$, which is analytic at $z=0$. Moreover, if $n_{r} \geq 2$, then (14) gives a representation of multiple zeta values as iterated integrals, namely

$$
\zeta\left(n_{1}, \ldots, n_{r}\right)=\int_{0}^{1} \omega_{0}^{n_{r}-1} \omega_{1} \ldots \omega_{0}^{n_{1}-1} \omega_{1} .
$$

The integral representation shows also that multiple polylogarithms can be extended to multi-valued functions on $\mathbb{P}^{1} \backslash\{0,1, \infty\}$. For many more properties of multiple polylogarithms, see for example the lecture notes [10].

\subsection{The Drinfeld associator}

Now we let $\mathbb{C}^{\prime}$ be the set of complex numbers with the two half-lines $(-\infty, 0]$ and $[1, \infty)$ removed. There exists a unique solution $G_{0}: \mathbb{C}^{\prime} \rightarrow \mathbb{C}\langle\langle x, y\rangle\rangle$ to the KnizhnikZamolodchikov equation (KZ-equation for short) 


$$
\frac{\partial}{\partial z} g(z)=\left(\frac{x}{z}+\frac{y}{z-1}\right) \cdot g(z)
$$

which satisfies $G_{0}(z) \sim z^{x}$ as $z \rightarrow 0$ on $\mathbb{C}^{\prime}$ [20]. Similarly, there exists a unique solution $G_{1}: \mathbb{C}^{\prime} \rightarrow \mathbb{C}\langle\langle x, y\rangle\rangle$ to the KZ-equation such that $G_{1}(z) \sim(1-z)^{y}$ as $z \rightarrow 1$ on $\mathbb{C}^{\prime}$. The quotient

$$
\Phi(x, y)=G_{1}^{-1}(z) G_{0}(z) \in \mathbb{C}\langle\langle x, y\rangle\rangle,
$$

which does not depend on $z$, is called the Drinfeld associator. An explicit formula in terms of iterated integrals can be given as follows (cf. [41], Section 2). Let $\Omega_{\mathrm{KZ}}$ denote the $\mathbb{C}\langle\langle x, y\rangle\rangle$-valued one-form given by

$$
\Omega_{K Z}=\omega_{0} \cdot x-\omega_{1} \cdot y .
$$

Then

$$
\Phi(x, y)=\lim _{t \rightarrow 0} e^{-\log (t) y} \exp \left[\int_{t}^{1-t} \Omega_{K Z}\right] e^{\log (t) x},
$$

where the iterated integration is performed along the straight-line path from $t$ to $1-t$, and exp was defined in (11). The coefficient of the word $x^{n_{r}-1} y \ldots x^{n_{1}-1} y$ in (19) is given by $(-1)^{r} \zeta\left(n_{1}, \ldots, n_{r}\right)$, as can be seen from the integral representation of multiple zeta values (15).

\section{Multiple elliptic polylogarithms and the elliptic KZB associator}

Both multiple polylogarithms and the Drinfeld associator possess elliptic analogues. In the case of polylogarithms, one obtains (multiple) elliptic polylogarithms as functions on the once-punctured elliptic curve $E_{\tau}^{\times} \cong\left(\mathbb{C}^{*} / q^{\mathbb{Z}}\right) \backslash\{1\}$ by averaging the ordinary polylogarithms along the spiral $q^{\mathbb{Z}}$, where $q=e^{2 \pi i \tau}$ with $\tau$ in the upper halfplane. This definition of elliptic polylogarithms was pioneered by Bloch [3] in the case of the single-valued dilogarithm, and later extended by Zagier [43] to all singlevalued polylogarithms. Finally, Brown and Levin treated the case of multi-valued multiple polylogarithms [15]. Furthermore, they also established a representation of multiple elliptic polylogarithms as homotopy invariant iterated integrals, thus paralleling the dichotomy between the sum and the integral representation of multiple polylogarithms (cf. Section 2.2). While the integrands for multiple zeta values were given by the Knizhnik-Zamolodchikov equation, the integrands of the multiple elliptic polylogarithms are constructed from the elliptic Knizhnik-ZamolodchikovBernard (KZB) differential equation [16, 32].

On the other hand, Enriquez introduced the notion of an elliptic associator in the context of an elliptic version of Drinfeld's version of Grothendieck-Teichmüller theory [21]. Furthermore, he constructs an explicit elliptic associator $(\Phi, A(\tau), B(\tau))$ from the regularized monodromy of the KZB equation. Here, $\Phi$ denotes the Drinfeld associator, and $A(\tau), B(\tau)$ are certain group-like elements of $\exp \left(\widehat{\mathfrak{t}}_{1,2}\right)$ (where 
$\widehat{\mathfrak{t}}$ denotes the completion of the elliptic braid Lie algebra on two strands), depending holomorphically on the coordinate $\tau$ in the upper half-plane. The group-likeness implies that the coefficients of the series $A(\tau)$ and $B(\tau)$ give rise to $\mathbb{Q}$-algebras $\mathscr{E} \mathscr{Z}^{\mathrm{A}}$ and $\mathscr{E} \mathscr{Z}^{\mathrm{B}}$, which are called the algebra of A-elliptic multiple zeta values and $B$-elliptic multiple zeta values respectively.

\subsection{An elliptic analogue of the KZ-equation}

The starting point for the construction of both multiple elliptic polylogarithms and the elliptic KZB associator is an elliptic analogue of the KZ-equation (16), namely the universal elliptic KZB equation [16,32]. It is defined by a connection on a certain vector bundle over the universal elliptic curve. We will consider only its restriction to a fiber of the universal elliptic curve, and moreover work with a certain real analytic trivialization of this restricted bundle, which was introduced in [15].

\subsubsection{A meromorphic Jacobi form}

Let $\mathbb{H}=\{z \in \mathbb{C} \mid \mathfrak{I}(z)>0\}$ denote the upper-half plane, and fix a point $\tau \in \mathbb{H}$. We consider the following Jacobi theta function

$$
\theta_{\tau}(\xi)=\sum_{n \in \mathbb{Z}}(-1)^{n} q^{\frac{1}{2}\left(n+\frac{1}{2}\right)^{2}} e^{\left(n+\frac{1}{2}\right) 2 \pi i \xi}, \quad q=e^{2 \pi i \tau} .
$$

Definition 1. We define a meromorphic function on $\mathbb{C} \times \mathbb{C}$ by the formula

$$
F_{\tau}(\xi, \alpha)=\frac{\theta_{\tau}^{\prime}(0) \theta_{\tau}(\xi+\alpha)}{\theta_{\tau}(\xi) \theta_{\tau}(\alpha)} .
$$

Following [15], we call $F_{\tau}(\xi, \alpha)$ the Kronecker function, [42]. The terminology for (21) varies in the literature. In [44], it is called "a meromorphic Jacobi form", while in [1], it is referred to as "the Kronecker theta function".

Since $F_{\tau}$ is meromorphic, it has a Laurent expansion in $\alpha$. In what follows, we will consider $F_{\tau}(\xi, \alpha)$ as a formal series in $\alpha$, whose coefficients are functions in $\xi$ (with $\tau$ being fixed). Note that the Kronecker series has simple poles for $\xi \in \mathbb{Z}+\mathbb{Z} \tau$, and is holomorphic outside of that lattice.

\subsubsection{Differential forms on a punctured elliptic curve}

Now consider the complex elliptic curve $E_{\tau}=\mathbb{C} /(\mathbb{Z}+\mathbb{Z} \tau)$ with canonical coordinate $\xi=s+r \tau$, where $r, s \in \mathbb{R}$. Write $E_{\tau}^{\times}$for $E_{\tau}$ with the origin 0 removed. Since the Kronecker series is quasi-periodic 


$$
F_{\tau}(\xi+1, \alpha)=F_{\tau}(\xi, \alpha), \quad F_{\tau}(\xi+\tau, w)=e^{-2 \pi i \frac{\mathfrak{I}(\xi)}{\mathfrak{I}(\tau)} \alpha} F_{\tau}(\xi, \alpha),
$$

(cf. [15], Proposition 5), the smooth differential one-form (where $\alpha$ is viewed as a formal variable)

$$
\Omega_{\tau}(\xi, \alpha)=e^{2 \pi i r \alpha} F_{\tau}(\xi, \alpha) d \xi
$$

descends to $E_{\tau}^{\times}$.

Definition 2 (Brown-Levin). Define a family $\left\{\omega^{(k)}\right\}_{k \geq 0}$ of real analytic, differential one-forms on $E_{\tau}^{\times}$by

$$
\Omega_{\tau}(\xi, \alpha)=\sum_{k \geq 0} \omega^{(k)} \alpha^{k-1}
$$

\subsubsection{The elliptic KZB equation}

With the differential form $\Omega_{\tau}(\xi, \alpha)$ in hand, we can now write down the elliptic KZB equation, following [15], Proposition 23. It is defined by a differential form $J_{\mathrm{KZB}}$, which takes values in the lower central series completion $\widehat{\mathbb{L}}_{\mathbb{C}}(x, y)$ of the free Lie algebra $\mathbb{L}_{\mathbb{C}}(x, y)$ over $\mathbb{C}$, which we will now describe. Let $U \subset \mathbb{C}$ be an open subset.

Definition 3. For a function $g: U \rightarrow \mathbb{C}$, the elliptic $K Z B$ equation is the differential equation

$$
d g(\xi)=J_{\mathrm{KZB}} \cdot g(\xi)
$$

with

$$
J_{\mathrm{KZB}}=-2 \pi i d r \cdot x+\operatorname{ad}(x) \Omega_{\tau}(\xi, \operatorname{ad}(x))(y) .
$$

An important property of the form $\omega_{\mathrm{KZB}}$ is that it satisfies the flatness condition

$$
d J_{\mathrm{KZB}}+J_{\mathrm{KZB}} \wedge J_{\mathrm{KZB}}=0
$$

which is straightforward to verify.

\subsection{The elliptic KZB associator}

Having described an elliptic analogue of multiple polylogarithms, we now turn to an elliptic analogue of the Drinfeld associator, the elliptic KZB associator [21]. Again, fix $\tau \in \mathbb{H}$. The starting point for the definition of the elliptic KZB associator is also the elliptic KZB equation

$$
d g(\xi)=-J_{\mathrm{KZB}} \cdot g(\xi)
$$

here with an additional minus sign, where $g: S \rightarrow \mathbb{C}\langle\langle x, y\rangle\rangle$ is defined on the simply connected domain $S=\{u+v i \in \mathbb{C} \mid u, v \in(0,1)\} \subset \mathbb{C}$. This equation has a unique 
solution $G$ defined on $S$, which satisfies $G(\xi) \sim(-2 \pi i \xi)^{-\operatorname{ad}(x)(y)}$, where the branch of the logarithm is chosen such that $\log ( \pm i)= \pm \frac{\pi i}{2}$. The following definition can be found in [21], Section 5 .

Definition 4 (Enriquez). The elliptic KZB associator is the triple $(\Phi, A(\tau), B(\tau))$, where $\Phi$ denotes the Drinfeld associator and $A(\tau), B(\tau) \in \mathbb{C}\langle\langle x, y\rangle\rangle$ are formal series, defined by the formulae

$$
A(\tau)=G(\xi)^{-1} G(\xi+1), \quad B(\tau)=G(\xi)^{-1} G(\xi+\tau) .
$$

By the same reasoning as for the Drinfeld associator, neither $A(\tau)$ nor $B(\tau)$ depend on the variable $\xi$. Since $G(\xi)$ can be expressed as an iterated integral

$$
G(\xi)=\lim _{\varepsilon \rightarrow 0} \exp \left[\int_{\xi}^{\varepsilon} J_{\mathrm{KZB}}\right](-2 \pi i \varepsilon)^{-\mathrm{ad}(x)(y)},
$$

one obtains explicit formulae for $A(\tau)$ and $B(\tau)$

$$
\begin{aligned}
& A(\tau)=e^{\pi i \mathrm{ad}(x)(y)} \lim _{\varepsilon \rightarrow 0}(-2 \pi i \varepsilon)^{\mathrm{ad}(x)(y)} \exp \left[\int_{\varepsilon}^{1-\varepsilon}-J_{\mathrm{KZB}}^{o p}\right](-2 \pi i \varepsilon)^{-\mathrm{ad}(x)(y)}, \\
& B(\tau)=e^{-\pi i \operatorname{ad}(x)(y)} \lim _{\varepsilon \rightarrow 0}(-2 \pi i \varepsilon)^{\operatorname{ad}(x)(y)} \exp \left[\int_{\varepsilon \tau}^{(1-\varepsilon) \tau}-J_{\mathrm{KZB}}^{o p}\right](-2 \pi i \varepsilon)^{-\mathrm{ad}(x)(y)} .
\end{aligned}
$$

where $J_{\mathrm{KZB}}$ is now considered as a $\mathbb{C}\langle\langle x, y\rangle\rangle^{o p}$-valued formal differential form, i.e. multiplication of words in $x, y$ has been reversed.

The series $A(\tau)$ and $B(\tau)$ have a number of interesting properties, first found by Enriquez. They satisfy a number of functional equations, which relate them to elliptic braid Lie algebras, and as $\tau \rightarrow i \infty$, they degenerate to the Drinfeld associator. For all these properties and much more on elliptic associators in general, see [21].

\subsection{Multiple elliptic polylogarithms}

Multiple elliptic polylogarithms give a second perspective on elliptic multiple zeta values.

\subsubsection{Series representation}

Roughly speaking, multiple elliptic polylogarithms are obtained from multiple polylogarithms by averaging along the spiral $q^{\mathbb{Z}}$, where $q=e^{2 \pi i \tau}$. This uses the Jacobi uniformization $E_{\tau} \cong \mathbb{C}^{*} / q^{\mathbb{Z}}$ given by $\xi \mapsto z=e^{2 \pi i \xi}$, and yields functions of the schematic form

$$
\sum_{n \in \mathbb{Z}} \operatorname{Li}_{n_{1}, \ldots, n_{r}}\left(q^{n} z\right)
$$


In order to make this approach rigorous, one has to employ a delicate regularization process, which in particular requires extensive knowledge about the singularities of (multi-variable) multiple polylogarithms. We cannot give the technical details here, and refer instead to Sections 6 and 7 of [15].

\subsubsection{Integral representation}

In [15], one also finds an approach to multiple elliptic polylogarithms via iterated integrals. Consider the formal series of iterated integrals on $E_{\tau}^{\times}$

$$
T=\exp \left[\int J_{\mathrm{KZB}}\right] \in \operatorname{Hom}\left(P E_{\tau}^{\times}, \widehat{\mathbb{L}}_{\mathbb{C}}(x, y)\right),
$$

where $\operatorname{Hom}\left(P E_{\tau}^{\times}, \mathbb{C}\right)$ denotes the set of complex-valued functions on the set $P E_{\tau}^{\times}$ of piecewise smooth paths on $E_{\tau}^{\times}$, and the tensor product is completed. The flatness of (28) implies that in fact every coefficient of $T$, viewed as a power series in $x, y$, is a homotopy invariant iterated integral. One of the main results of [15] is:

Theorem 2 (Brown-Levin). Every homotopy invariant iterated integral on $E_{\tau}^{\times}$ arises as a $\mathbb{C}$-linear combination of coefficients of $T$. Moreover, the $\mathbb{Q}$-vector space spanned by the coefficients of $T$ is equal to the $\mathbb{Q}$-vector space spanned by the multiple elliptic polylogarithms, as subspaces of the $\mathbb{Q}$-vector space of multi-valued functions on $E_{\tau}^{\times}$.

In this way, one obtains a complete description of multiple elliptic polylogarithms in terms of homotopy invariant iterated integrals on a once-punctured complex elliptic curve, which is the elliptic analogue of the iterated integral representation of the classical multiple polylogarithms (14).

\subsubsection{Elliptic associators via elliptic polylogarithms}

The relation between [15] and the work of Enriquez is established by the fact that the series $A(\tau)$ and $B(\tau)$ are restrictions of (35) to certain natural paths on $E_{\tau}^{\times}$. This follows from the explicit description of the KZB-associator (32), (33). The construction of the elliptic KZB-associator using the function $T$ has the advantage that elliptic multiple zeta values are exhibited as restrictions of homotopy invariant iterated integrals, which is a necessary prerequisite for any sort of relation to unipotent fundamental groups [18]. It also tightens the analogy with multiple zeta values, which are given by homotopy invariant iterated integrals on $\mathbb{P}^{1} \backslash\{0,1, \infty\}$. 


\section{The algebra of A-elliptic multiple zeta values}

In the last section, we have given a very short introduction to multiple elliptic polylogarithms and the elliptic KZB associator, indicating how the two objects are related. On the other hand, in Section 2, we saw that multiple zeta values can be defined as special values of multiple polylogarithms, or equivalently as coefficients of the Drinfeld associator. In light of this fact we are lead to a definition of elliptic analogues of multiple zeta values as special values of multiple elliptic polylogarithms or as coefficients of the series $A(\tau)$ and $B(\tau)$. However, we proceed slightly differently, first giving a direct definition of A-elliptic multiple zeta values as iterated integrals $I^{\mathrm{A}}\left(n_{1}, \ldots, n_{r} ; \tau\right)$ over the differential forms $\omega^{(k)}(25)$. That this $\mathbb{Q}$-vector space of iterated integrals is equal to the $\mathbb{Q}$-vector space spanned by the coefficients of (a slight variation of) the series $A(\tau)$ was shown in [36].

\subsection{Definition of A-elliptic multiple zeta values}

Definition 5. We define $I^{\mathrm{A}}\left(n_{1}, \ldots, n_{r} ; \tau\right)$ to be the shuffle-regularized ${ }^{2}$ iterated integral

$$
I^{\mathrm{A}}\left(n_{1}, \ldots, n_{r} ; \tau\right)=\int_{\alpha} \omega^{\left(n_{r}\right)} \ldots \omega^{\left(n_{1}\right)}
$$

We call $I^{\mathrm{A}}\left(n_{1}, \ldots, n_{r} ; \tau\right)$ an A-elliptic multiple zeta value. It is said to have weight $n_{1}+\ldots+n_{r}$ and length $r$. The $\mathbb{Q}$-vector space $\mathscr{E} \mathscr{Z}^{\mathrm{A}}$ spanned by the $I^{\mathrm{A}}\left(n_{1}, \ldots, n_{r} ; \tau\right)$ will be called the space of A-elliptic multiple zeta values.

By the shuffle product formula for iterated integrals, it follows that $\mathscr{E} \mathscr{Z}^{\mathrm{A}}$ is a $\mathbb{Q}$ subalgebra of the $\mathbb{C}$-algebra of holomorphic functions $\mathscr{O}(\mathbb{H})$ on $\mathbb{H}$. In particular, the rational number $1 \in \mathbb{Q}$ is an A-elliptic multiple zeta value of weight and length equal to zero, in accordance with our conventions for the empty iterated integral.

It is known that the algebra of multiple zeta values is also the $\mathbb{Q}$-vector space spanned by the coefficients of the Drinfeld associator. There is an analogous result for A-elliptic multiple zeta values. Write

$$
\widetilde{A}(\tau)=e^{-\pi i \operatorname{ad}(x)(y)} A(\tau)=\sum_{w \in\langle x, y\rangle} \widetilde{A}(\tau)_{w} \cdot w .
$$

Note that the constant pre-factor $e^{-\pi i \operatorname{ad}(x)(y)}$, which is multiplied to $A(\tau)$, is an artifact of the difference between the regularization procedure for the KZB-associator, and the regularization of the iterated integrals (35).

Proposition 1. We have an equality of vector spaces

$$
\mathscr{E} \mathscr{Z}^{\mathrm{A}}=\left\langle\widetilde{A}(\tau)_{w} \mid w \in\langle x, y\rangle\right\rangle_{\mathbb{Q}} .
$$

${ }^{2}$ Iterated integrals starting or ending with $\omega^{(1)}$ diverge, and need to be regularized, such that the shuffle product formula remains valid. See $[22,36]$ for details on this regularization procedure. 
Proof. See [36], Proposition 2.3.

\subsection{Relations between A-elliptic multiple zeta values}

Recall that A-elliptic multiple zeta values are defined as iterated integrals over the differential forms $\omega^{(k)}$, which in turn are the coefficients of the Kronecker differential form

$$
\sum_{k \geq 0} \omega^{(k)} \alpha^{k-1}=\Omega_{\tau}(\xi, \alpha)=e^{2 \pi i r \alpha} \frac{\theta_{\tau}^{\prime}(0) \theta_{\tau}(\xi+\alpha)}{\theta_{\tau}(\xi) \theta_{\tau}(\alpha)} d \xi
$$

where $\xi=s+r \tau$.

\subsubsection{Shuffle relations}

From (9), one deduces a shuffle product formula for A-elliptic multiple zeta values, which is conveniently expressed using the generating series

$$
\mathscr{I}^{\mathrm{A}}\left(X_{1}, X_{2}, \ldots, X_{r} ; \tau\right)=\sum_{n_{1}, \ldots, n_{r}=0}^{\infty} I^{\mathrm{A}}\left(n_{1}, \ldots, n_{r} ; \tau\right) X_{1}^{n_{1}-1} X_{2}^{n_{2}-1} \ldots X_{r}^{n_{r}-1}
$$

of A-elliptic multiple zeta values of length $r$, for $r \geq 0$. Explicitly, we have

$$
\mathscr{I}^{\mathrm{A}}\left(X_{1}, \ldots, X_{l} ; \tau\right) \mathscr{I}^{\mathrm{A}}\left(X_{l+1}, \ldots, X_{n} ; \tau\right)=\sum_{\sigma \in \Sigma_{l, n-l}} \mathscr{I}^{\mathrm{A}}\left(X_{\sigma^{-1}(1)}, \ldots, X_{\sigma^{-1}(n)} ; \tau\right),
$$

where $\Sigma_{l, n-l}$ denotes the set of $(l, n-l)$-shuffles. For example, in small lengths we get

$$
\begin{aligned}
\mathscr{I}^{\mathrm{A}}\left(X_{1} ; \tau\right) \mathscr{I}^{\mathrm{A}}\left(X_{2} ; \tau\right)=\mathscr{I}^{\mathrm{A}}\left(X_{1}, X_{2} ; \tau\right) & +\mathscr{I}^{\mathrm{A}}\left(X_{2}, X_{1} ; \tau\right), \\
\mathscr{I}^{\mathrm{A}}\left(X_{1} ; \tau\right) \mathscr{I}^{\mathrm{A}}\left(X_{2}, X_{3} ; \tau\right)=\mathscr{I}^{\mathrm{A}}\left(X_{1}, X_{2}, X_{3} ; \tau\right) & +\mathscr{I}^{\mathrm{A}}\left(X_{2}, X_{1}, X_{3} ; \tau\right) \\
& +\mathscr{I}^{\mathrm{A}}\left(X_{2}, X_{3}, X_{1} ; \tau\right) .
\end{aligned}
$$

\subsubsection{Reflection relations}

Further relations between A-elliptic multiple zeta values can be inferred from functional equations for $\Omega_{\tau}$, for example, the symmetry equation (cf. [44], Theorem.(i) in Section 3)

$$
\Omega_{\tau}(-\xi,-\alpha)=\Omega_{\tau}(\xi, \alpha) .
$$

Writing $\omega^{(k)}=f^{(k)} d \xi$, for some real-analytic function $f^{(k)}: E_{\tau}^{\times} \rightarrow \mathbb{C}$, (44) implies

$$
f^{(k)}(-\xi)=(-1)^{k} f^{(k)}(\xi)
$$


Using (45) and the reversal of paths formula for iterated integrals, one obtains the reflection relation for A-elliptic multiple zeta values (cf. [6], eq. (2.13))

$$
I^{\mathrm{A}}\left(n_{1}, \ldots, n_{r} ; \tau\right)=(-1)^{n_{1}+\ldots+n_{r}} I^{\mathrm{A}}\left(n_{r}, \ldots, n_{1} ; \tau\right),
$$

which, on the level of generating series, simply becomes the functional equation

$$
\mathscr{I}^{\mathrm{A}}\left(X_{1}, \ldots, X_{r} ; \tau\right)=\mathscr{I}^{\mathrm{A}}\left(-X_{r}, \ldots,-X_{1} ; \tau\right) .
$$

\subsubsection{Fay relations}

The second, more interesting, functional equation satisfied by $\Omega_{\tau}$ is the Fay identity (cf. [15], Proposition 4)

$$
\begin{aligned}
\Omega_{\tau}\left(\xi_{1}, \alpha_{1}\right) \wedge \Omega_{\tau}\left(\xi_{2}, \alpha_{2}\right) & =\Omega_{\tau}\left(\xi_{1}-\xi_{2}, \alpha_{1}\right) \wedge \Omega_{\tau}\left(\xi_{2}, \alpha_{1}+\alpha_{2}\right) \\
& +\Omega_{\tau}\left(\xi_{2}-\xi_{1}, \alpha_{2}\right) \wedge \Omega_{\tau}\left(\xi_{1}, \alpha_{1}+\alpha_{2}\right) .
\end{aligned}
$$

The Fay identity for $\Omega_{\tau}(\xi, \alpha)$ implies quadratic relations for the functions $f^{(k)}$, namely

$$
\begin{aligned}
f^{(m)}\left(\xi_{1}\right) f^{(n)}\left(\xi_{2}\right)= & -(-1)^{n} f^{(m+n)}\left(\xi_{1}-\xi_{2}\right) \\
& +\sum_{r=0}^{n}\left(\begin{array}{c}
m+r-1 \\
m-1
\end{array}\right) f^{(n-r)}\left(\xi_{2}-\xi_{1}\right) f^{(m+r)}\left(\xi_{1}\right) \\
& +\sum_{r=0}^{m}\left(\begin{array}{c}
n+r-1 \\
n-1
\end{array}\right) f^{(m-r)}\left(\xi_{1}-\xi_{2}\right) f^{(n+r)}\left(\xi_{2}\right) .
\end{aligned}
$$

The Fay identity also yields $\mathbb{Q}$-linear relations between A-elliptic multiple zeta values, which are again most conveniently expressed as functional equations for the generating series $\mathscr{I}^{\mathrm{A}}$. In length two, we have (cf. [36], eq. (2.40))

$$
\mathscr{I}^{\mathrm{A}}(X, Y ; \tau)+\mathscr{I}^{\mathrm{A}}(X+Y,-Y ; \tau)+\mathscr{I}^{\mathrm{A}}(-X-Y, X ; \tau)=3 \zeta(2) .
$$

Observe that the right hand side of (50) corresponds to a subset of divergent Aelliptic multiple zeta values, such as $I^{\mathrm{A}}(1,1 ; \tau)$, which require a regularization procedure. While A-elliptic multiple zeta values have been regularized in a way compatible with the shuffle product, one can ask whether there is a "Fay-regularization" for a priori divergent A-elliptic multiple zeta values. In a similar vein, note that the stuffle regularization for multiple zeta values differs from the shuffle regularization, and that there is an explicit formula for the difference between the two regularizations (cf. [38], Proposition 2.4.14). For A-elliptic multiple zeta values, for example, the first equation in (50) suggests the convention

$$
\mathscr{I}_{*}^{\mathrm{A}}(X, Y ; \tau):=\mathscr{I}^{\mathrm{A}}(X, Y ; \tau)-\zeta(2)=\mathscr{I}^{\mathrm{A}}(X, Y ; \tau)+\frac{1}{2} I^{\mathrm{A}}(2 ; \tau),
$$


such that $\mathscr{I}_{*}^{\mathrm{A}}(X, Y ; \tau)+\mathscr{I}_{*}^{\mathrm{A}}(X+Y,-Y ; \tau)+\mathscr{I}_{*}^{\mathrm{A}}(-X-Y, X ; \tau)=0$. See also [6], Section 2.2, for Fay relations in higher lengths.

\subsection{The dimension of the space of A-elliptic multiple zeta values}

Now that we have seen that there are many relations between A-elliptic multiple zeta values, it is a natural next step to try and count these relations, in order to get upper bounds on the dimensions of the space of A-elliptic multiple zeta values. To arrive at a more precise formulation, consider for non-negative integers $N, l$ the $\mathbb{Q}$-vector subspace

$$
\mathscr{L}_{l}\left(\mathscr{E} \mathscr{Z}_{N}^{\mathrm{A}}\right)=\left\langle I^{\mathrm{A}}\left(n_{1}, \ldots, n_{r} ; \tau\right) \in \mathscr{E} \mathscr{Z}^{\mathrm{A}} \mid n_{1}+\ldots+n_{r}=N, r \leq l\right\rangle_{\mathbb{Q}} \subset \mathscr{E} \mathscr{Z}^{\mathrm{A}},
$$

spanned by A-elliptic multiple zeta values of weight $N$ and length at most $l$. From (41), it is clear that the length filtration is compatible with the algebra structure of $\mathscr{E} \mathscr{Z}^{\mathrm{A}}$, and that therefore $\mathscr{E} \mathscr{Z}^{\mathrm{A}}$ is a filtered $\mathbb{Q}$-algebra. Denote by $\operatorname{gr}_{l}^{\mathscr{L}}\left(\mathscr{E} \mathscr{Z}_{N}^{\mathrm{A}}\right)=$ $\mathscr{L}_{l}\left(\mathscr{E} \mathscr{Z}_{N}^{\mathrm{A}}\right) / \mathscr{L}_{l-1}\left(\mathscr{E} \mathscr{Z}_{N}^{\mathrm{A}}\right)$ the associated graded. Since there are only finitely many A-elliptic multiple zeta values of a given weight and length, $\operatorname{gr}_{l}^{\mathscr{L}}\left(\mathscr{E} \mathscr{Z}_{N}^{\mathrm{A}}\right)$ is a finitedimensional $\mathbb{Q}$-vector space, and as outlined in Problem 1, we would like to compute the dimension

$$
D_{N, l}^{\mathrm{ell}}=\operatorname{dim}_{\mathbb{Q}} \mathrm{gr}_{l}^{\mathscr{L}}\left(\mathscr{E} \mathscr{Z}_{N}^{\mathrm{A}}\right)
$$

for all $N, l \geq 0$.

The case $l=0$ is of course trivial: we have $D_{N, 0}^{\text {ell }}=\delta_{N, 0}$, since the empty iterated integral $\int_{\gamma}=1$ has weight zero, by definition. In the cases $l=1,2$, a complete solution to Problem 1 has been obtained in [36], on which we will report briefly in the rest of this section.

\subsubsection{Elliptic zeta values}

We begin our investigation of Problem 1 in length $l=1$. It is clear from Definition 5 that for a given weight $N$, there is only one A-elliptic multiple zeta value of length one, namely $I^{\mathrm{A}}(N ; \tau)$. Thus $D_{N, 1}^{\mathrm{ell}} \leq 1$.

Now by (46), we have

$$
I^{\mathrm{A}}(N ; \tau)=(-1)^{N} I^{\mathrm{A}}(N ; \tau),
$$

which immediately yields $I^{\mathrm{A}}(N ; \tau)=0$, if $N$ is odd, hence $D_{N, 1}^{\text {ell }}=0$ in that case. In Section 5.4, we will see that for even $N \geq 0$

$$
I^{\mathrm{A}}(N ; \tau)=-2 \zeta(N),
$$

and we obtain a complete solution of Problem 1 in the length one case 


$$
D_{N, 1}^{\text {ell }}= \begin{cases}0 & N=0 \text { or } N \text { odd } \\ 1 & \text { else. }\end{cases}
$$

Also, note that since the even zeta values $\zeta(2 n)$ are linearly independent over $\mathbb{Q}$, it follows that the space of elliptic zeta values $\mathscr{L}_{1}\left(\mathscr{E} \mathscr{Z}^{\mathrm{A}}\right)=\sum_{N \geq 0} \mathscr{L}_{1}\left(\mathscr{E} \mathscr{Z}_{N}^{\mathrm{A}}\right)$ is graded for the weight, i.e.

$$
\mathscr{L}_{1}\left(\mathscr{E} \mathscr{Z}^{\mathrm{A}}\right)=\bigoplus_{N \geq 0} \mathscr{L}_{1}\left(\mathscr{E} \mathscr{Z}_{N}^{\mathrm{A}}\right)
$$

\subsubsection{Elliptic double zeta values of even weight}

We now investigate the case $l=2$ and $N$ even, i.e. A-elliptic multiple zeta values of length two and even weight. From (42) together with (55) and the fact that $\zeta(N) \in$ $\left\langle(2 \pi i)^{N}\right\rangle_{\mathbb{Q}}$, if $N$ is even, we know that

$I^{\mathrm{A}}\left(n_{1}, n_{2} ; \tau\right)+I^{\mathrm{A}}\left(n_{2}, n_{1} ; \tau\right)=I^{\mathrm{A}}\left(n_{1} ; \tau\right) I^{\mathrm{A}}\left(n_{2} ; \tau\right) \in\left\langle(2 \pi i)^{n_{1}+n_{2}}\right\rangle_{\mathbb{Q}} \subset \mathscr{L}_{1}\left(\mathscr{E} \mathscr{Z}_{n_{1}+n_{2}}^{\mathrm{A}}\right)$.

On the other hand, if the weight $N=n_{1}+n_{2}$ is even, then it follows from (46) that

$$
I^{\mathrm{A}}\left(n_{1}, n_{2} ; \tau\right)+I^{\mathrm{A}}\left(n_{2}, n_{1} ; \tau\right)=2 I^{\mathrm{A}}\left(n_{1}, n_{2} ; \tau\right),
$$

and therefore in that case, by (55),

$$
I^{\mathrm{A}}\left(n_{1}, n_{2} ; \tau\right)= \begin{cases}0 & n_{1}, n_{2} \text { odd } \\ 2 \zeta\left(n_{1}\right) \zeta\left(n_{2}\right) & n_{1}, n_{2} \text { even. }\end{cases}
$$

In particular, $D_{N, 2}^{\text {ell }}=0$, if $N$ is even.

\subsubsection{Elliptic double zeta values of odd weight}

So far, we have seen that both the elliptic zeta values $I^{\mathrm{A}}(n ; \tau)$, as well as the elliptic double zeta values $I^{\mathrm{A}}\left(n_{1}, n_{2} ; \tau\right)$ of even weight are constant as functions in $\tau$, and that they are equal to rational multiples of powers of $(2 \pi i)^{N}$, where $N$ is the weight. If the weight is odd, this is no longer true, since in that case (46) gives no new information about elliptic double zeta values of odd weight. Indeed, since the product of two elliptic zeta values of weights of different parities necessarily vanishes, the shuffle product formula becomes

$$
I^{\mathrm{A}}\left(n_{1}, n_{2} ; \tau\right)+I^{\mathrm{A}}\left(n_{2}, n_{1} ; \tau\right)=0
$$

which is also precisely the reflection relation in the case of odd weight.

In order to attack Problem 1 in the odd weight case, we first count the number of linearly independent Fay and shuffle relations. For this, we denote by $\widehat{V}_{N} \subset \mathbb{Q}(X, Y)$ 
the $\mathbb{Q}$-vector space of rational functions $P(X, Y)$ in the variables $X$ and $Y$, such that the product $X Y \cdot P$ is a homogeneous polynomial of degree $N+2$.

Definition 6. We define the length two Fay-shuffle space $\mathrm{FSh}_{2}(N)$ of weight $N$ to be the subspace $\mathrm{FSh}_{2}(N) \subset \widehat{V}_{N}$ of elements, satisfying the Fay and shuffle equations

$$
P(X, Y)+P(X+Y,-Y)+P(-X-Y, X)=0, \quad P(X, Y)+P(Y, X)=0 .
$$

In particular, since in the length two case the right hand side of (50) and the left hand side of (42) vanish in $\operatorname{gr}_{2}^{\mathscr{L}}\left(\mathscr{E} \mathscr{Z}^{\mathrm{A}}\right)$, as both are contained in $\mathscr{L}_{1}\left(\mathscr{E} \mathscr{Z}^{\mathrm{A}}\right)$ by $\left.(55)\right)$, we see that the elliptic double zeta values satisfy the defining equations of $\mathrm{FSh}_{2}(N)$, modulo elliptic zeta values. As a consequence, we have:

Proposition 2. ([36], Proposition 3.10) The dimension of the Fay-shuffle space gives an upper bound for $D_{N, 2}^{\mathrm{ell}}$, i.e.

$$
D_{N, 2}^{\mathrm{ell}} \leq \operatorname{dim}_{\mathbb{Q}} \mathrm{FSh}_{2}(N-2),
$$

for all $N \geq 0$.

The dimensions on the right hand side can in fact be computed using invariant theory.

Theorem 3. ([36], Theorem 3.11) We have

$$
\operatorname{dim}_{\mathbb{Q}} \operatorname{FSh}_{2}(N)= \begin{cases}0 & \text { if } N \text { is even } \\ \left\lfloor\frac{N}{3}\right\rfloor+1 & \text { if } N \text { is odd } .\end{cases}
$$

It remains to show that $D_{N, 2}^{\text {ell }} \geq\left\lfloor\frac{N}{3}\right\rfloor+1$ for odd $N$, and this follows from:

Theorem 4. ([36], Theorem 3.15) Let $N \geq 1$ be odd. The family of elliptic double zeta values

$$
\left\{I^{\mathrm{A}}(r, N-r ; \tau) \mid 0 \leq r \leq\lfloor N / 3\rfloor\right\}
$$

is linearly independent over $\mathbb{Q}$.

The proof uses an explicit representation of elliptic double zeta values as indefinite integrals of Eisenstein series (88). Using in addition the fact that there are no nontrivial relations between elliptic double zeta values of different weights (cf. [36], Theorem 3.6), the two preceding theorems also imply that every relation between elliptic double zeta values is a consequence of Fay and shuffle relations.

\section{Elliptic multiple zeta values and iterated Eisenstein integrals}

In this section, we will discuss the relation between A-elliptic multiple zeta values and indefinite iterated integrals of Eisenstein series [12,34]. This relation has first been established by Enriquez [21,22], who showed that the derivative of an 
A-elliptic multiple zeta value of length $r$ can be expressed using A-elliptic multiple zeta values of length $r-1$ and Eisenstein series. Moreover, the boundary condition for Enriquez's differential equation is given explicitly in terms of multiple zeta values. Using Enriquez's ideas as a starting point, one finds in [6] completely explicit formulae for A-elliptic multiple zeta values as linear combinations of iterated integrals of Eisenstein series and multiple zeta values. It turns out that the precise linear combinations of iterated Eisenstein integrals, which appear as elliptic multiple zeta values are controlled by a certain well-studied Lie algebra $\mathfrak{u}^{\text {geom }}$ of derivations of a free Lie algebra on two generators [13, 16, 32, 37].

\subsection{Reminder on iterated Eisenstein integrals}

For $k \geq 1$, define the Eisenstein series $G_{k}(\tau)$ to be

$$
G_{2 k}(\tau)=\sum_{(m, n) \in \mathbb{Z} \backslash\{(0,0)\}} \frac{1}{(m+n \tau)^{k}},
$$

where for $k=1,2$, we use Eisenstein summation for double series, i.e.

$$
\sum_{(m, n) \in \mathbb{Z} \backslash\{(0,0)\}} a_{m, n}=\lim _{N \rightarrow \infty} \lim _{M \rightarrow \infty} \sum_{n=-N}^{N} \sum_{m=-M}^{M} a_{m, n} .
$$

We also set $G_{0}(\tau) \equiv-1$. It is well-known that the Eisenstein series $G_{2 k}(\tau), k \geq 1$, can be expanded as a Fourier series in $q=e^{2 \pi i \tau}$

$$
G_{2 k}(\tau)=2 \zeta(2 k)+2 \frac{(2 \pi i)^{2 k}}{(2 k-1) !} \sum_{n \geq 1} \sigma_{2 k-1}(n) q^{n},
$$

where $\sigma_{k}(n)=\sum_{d \mid n} d^{k}$ denotes the sum of the $k$-th powers of the divisors of $n$.

In [12], one finds a definition of iterated integrals of Eisenstein series (and even of iterated integrals of more general modular forms, the so-called iterated Eichler or iterated Shimura integrals)

$$
\mathscr{G}\left(k_{1}, \ldots, k_{r} ; \tau\right)=\int_{\tau}^{i \infty} G_{k_{1}}\left(\tau_{1}\right) d \tau_{1} \ldots G_{k_{r}}\left(\tau_{r}\right) d \tau_{r},
$$

where the integral has to be regularized suitably at the boundary $i \infty$. Since the Eisenstein series are holomorphic functions on a one-dimensional complex manifold, the iterated integral (82) is independent of the choice of path from $\tau$ to $i \infty$. We refer to [12], Section 4, for the precise regularization scheme, which involves tangential base points, and confine ourselves with giving explicit formulae in lengths one and two: 


$$
\mathscr{G}(k ; \tau)=-\frac{1}{2 \pi i}\left(2 \zeta(k) \log q+\frac{2(2 \pi i)^{k}}{(k-1) !} \sum_{m, n=1}^{\infty} \frac{m^{k-2}}{n} q^{m n}\right),
$$

and

$$
\begin{aligned}
\mathscr{G}\left(k_{1}, k_{2} ; \tau\right)=\frac{1}{(2 \pi i)^{2}} & \left(2 \zeta\left(k_{1}\right) \zeta\left(k_{2}\right)(\log q)^{2}\right. \\
& +2 \zeta\left(k_{2}\right) \frac{2(2 \pi i)^{k_{1}}}{\left(k_{1}-1\right) !} \log q \sum_{m, n=1}^{\infty} \frac{m^{k_{1}-2}}{n} q^{m n} \\
& +2 \zeta\left(k_{1}\right) \frac{2(2 \pi i)^{k_{2}}}{\left(k_{2}-1\right) !} \sum_{m, n=1}^{\infty} \frac{m^{k_{2}-3}}{n^{2}} q^{m n} \\
& -2 \zeta\left(k_{2}\right) \frac{2(2 \pi i)^{k_{1}}}{\left(k_{1}-1\right) !} \sum_{m, n=1}^{\infty} \frac{m^{k_{1}-3}}{n^{2}} q^{m n} \\
& \left.+\frac{4(2 \pi i)^{k_{1}+k_{2}}}{\left(k_{1}-1\right) !\left(k_{2}-1\right) !} \sum_{m_{i}, n_{i}=1}^{\infty} \frac{m_{1}^{k_{1}-1} m_{2}^{k_{2}-2}}{\left(m_{1} n_{1}+m_{2} n_{2}\right) n_{2}} q^{m_{1} n_{1}+m_{2} n_{2}}\right),
\end{aligned}
$$

which both follow from [12] Example 4.10, and from (68). Note that

$$
\frac{\partial \mathscr{G}(k ; \tau)}{\partial \tau}=-G_{k}(\tau), \quad \frac{\partial \mathscr{G}\left(k_{1}, k_{2} ; \tau\right)}{\partial \tau}=-G_{k_{1}}(\tau) \mathscr{G}\left(k_{2} ; \tau\right),
$$

which can be verified directly, and generalizes to

$$
\frac{\partial \mathscr{G}\left(k_{1}, \ldots, k_{r} ; \tau\right)}{\partial \tau}=-G_{k_{1}}(\tau) \mathscr{G}\left(k_{2}, \ldots, k_{r} ; \tau\right),
$$

using a general property of iterated integrals (cf. [12], Proposition 4.7, for the case of iterated Eichler integrals).

\subsection{The differential equation for A-elliptic multiple zeta values}

As proved by Enriquez (cf. [22], Théorème 3.10), A-elliptic multiple zeta values satisfy a differential equation involving Eisenstein series. This differential equation is most conveniently written down in terms of the generating series $\mathscr{I}^{\mathrm{A}}\left(X_{1}, \ldots, X_{r} ; \tau\right)$ of A-elliptic multiple zeta values of length $r(40)$.

Theorem 5 (Enriquez). For all $r \geq 0$, we have 


$$
\begin{aligned}
2 \pi i \frac{\partial}{\partial \tau} \mathscr{I}^{\mathrm{A}}\left(X_{1}, \ldots, X_{r} ; \tau\right) & =\wp_{\tau}^{*}\left(X_{1}\right) \mathscr{I}^{\mathrm{A}}\left(X_{2}, \ldots, X_{r} ; \tau\right)-\wp_{\tau}^{*}\left(X_{r}\right) \mathscr{I}^{\mathrm{A}}\left(X_{1}, \ldots, X_{r-1} ; \tau\right) \\
& +\sum_{i=1}^{r-1}\left(\wp_{\tau}^{*}\left(X_{i+1}\right)-\wp_{\tau}^{*}\left(X_{i}\right)\right) \mathscr{I}^{\mathrm{A}}\left(X_{1}, \ldots, X_{i, i+1}, \ldots, X_{r} ; \tau\right),
\end{aligned}
$$

where $X_{i, j}:=X_{i}+X_{j}$ and $\wp_{\tau}^{*}(\alpha)=\sum_{k=0}^{\infty}(2 k-1) G_{2 k}(\tau) \alpha^{2 k-2}$.

Note that $\wp_{\tau}^{*}$ is related to the Weierstrass $\wp_{\tau}$-function by the formula $\wp_{\tau}^{*}(\alpha)=$ $\wp_{\tau}(\alpha)+G_{2}(\tau)$. The proof of Theorem 5 uses special properties of the Kronecker series, in particular that it satisfies the mixed heat equation (cf. [15], Proposition 4). The upshot is that the derivative of an A-elliptic multiple zeta value of length $r$ can be expressed using A-elliptic multiple zeta values of lengths $r-1$ and Eisenstein series. This in turn identifies A-elliptic multiple zeta values as iterated integrals of Eisenstein series of length $r-1$, up to a constant term, the reconstruction of which we turn to next.

\subsection{Restoring the constant terms of A-elliptic multiple zeta values}

By definition, the generating series of A-elliptic multiple zeta values is given by a version of Enriquez's A-associator [22, 36]

$$
\widetilde{A}(\tau)=\sum_{r \geq 0}(-1)^{r} \sum_{n_{1}, \ldots, n_{r} \geq 0} I^{\mathrm{A}}\left(n_{1}, \ldots, n_{r} ; \tau\right) \operatorname{ad}^{n_{r}}(x)(y) \ldots \mathrm{ad}^{n_{1}}(x)(y) .
$$

From [21], Proposition 5.4, it follows that $\widetilde{A}(\tau)$ satisfies $\widetilde{A}(\tau+1)=\widetilde{A}(\tau)$ and that it is holomorphic at infinity. Thus, it possesses a Fourier expansion in $q=e^{2 \pi i \tau}$, and the same is true for every A-elliptic multiple zeta value. Moreover, Enriquez has shown that the coefficients of the Fourier expansion of A-elliptic multiple zeta values are essentially given by multiple zeta values. We state this result as a proposition (cf. [22], Proposition 5.2).

Proposition 3 (Enriquez). Every A-elliptic multiple zeta values can be written as a Fourier series

$$
\sum_{n \geq 0} a_{n} q^{n}
$$

where $a_{n} \in \mathscr{Z}\left[(2 \pi i)^{-1}\right]$, and $\mathscr{Z} \subset \mathbb{R}$ denotes the $\mathbb{Q}$-algebra spanned by the multiple zeta values.

An immediate consequence of the last proposition is that the constant term $I_{0}^{\mathrm{A}}$ of an A-elliptic multiple zeta value can be retrieved as the $\operatorname{limit}_{\tau \rightarrow i \infty} I^{\mathrm{A}}\left(n_{1}, \ldots, n_{r} ; \tau\right)$. In order to compute this limit, we use the following result of Enriquez (cf. [22], eq. (7)).

Theorem 6 (Enriquez). The generating series of A-elliptic multiple zeta values has the following asymptotic behavior 


$$
\lim _{\tau \rightarrow i \infty} \widetilde{A}(\tau)=e^{\pi i t} \Phi(\tilde{y}, t) e^{2 \pi i \tilde{y}} \Phi(\tilde{y}, t)^{-1},
$$

where $t=-\operatorname{ad}(x)(y), \tilde{y}=-\frac{\operatorname{ad}(x)}{e^{2 \pi i \mathrm{ad}(x)}-1}(y)$ and $\Phi$ denotes the Drinfeld associator.

We end this section by giving a few examples of constant terms of A-elliptic multiple zeta values (cf. [6], Section 2.3). If all of the indices $n_{1}, \ldots, n_{r} \neq 1$, then a closed formula for the constant term $I_{0}^{\mathrm{A}}\left(n_{1}, \ldots, n_{r}\right)$ of $I^{\mathrm{A}}\left(n_{1}, \ldots, n_{r} ; \tau\right)$ is easy to give, since in that case, only the factor $e^{2 \pi i \tilde{y}}$ in (77) yields a non-trivial contribution, and therefore

$$
\left.I_{0}^{\mathrm{A}}\left(n_{1}, n_{2}, \ldots, n_{r}\right)\right|_{n_{i} \neq 1}=\left\{\begin{array}{cl}
0 & \text { at least one } n_{i} \text { is odd and all } n_{i} \neq 1 \\
\frac{1}{r !} \prod_{i=1}^{r}\left(-2 \zeta\left(n_{i}\right)\right) & \text { all } n_{i} \text { even. }
\end{array}\right.
$$

On the other hand, if several of the $n_{i}$ are equal to one, then the formulae become more cumbersome to write down, since in that case the extraction of the relevant terms from (77) combined with the translation from the $\{\tilde{y}, t\}$ alphabet (in which (77) is expressed) to the $x, y$ alphabet (which is used for A-elliptic multiple zeta values) requires many more steps. An implementation of this procedure, using Mathematica, yields for example

$$
I_{0}^{\mathrm{A}}(1,0)=-\frac{i \pi}{2}, \quad I_{0}^{\mathrm{A}}(1,0,0)=-\frac{i \pi}{4}, \quad I_{0}^{\mathrm{A}}(1,0,0,0)=-\frac{i \pi}{12}+\frac{\zeta(3)}{(2 \pi i)^{2}},
$$

which generalizes to

$$
I_{0}^{\mathrm{A}}(\underbrace{1,0, \ldots, 0}_{r})=-\frac{2 \pi i}{4(r-1) !}+\sum_{k=1}^{\lfloor r / 2\rfloor-1} \frac{1}{(r-(2 k+1)) !} \frac{\zeta(2 k+1)}{(2 \pi i)^{2 k}},
$$

and shows that every odd Riemann zeta value arises as the constant term of some linear combination of A-elliptic multiple zeta value. Also, multiple zeta values, which cannot be written as polynomials in Riemann zeta values are found to appear as constant terms of higher length A-elliptic multiple zeta values. Conjecturally, the first such multiple zeta value appears in weight 8 , for example $\zeta(3,5)$, and we have

$$
\begin{aligned}
I_{0}^{\mathrm{A}}(1,0,0,1,0,0,0,0,0)=\frac{1}{(2 \pi i)^{6}}( & -\zeta(3,5)-2 \zeta(2) \zeta(3)^{2}-\frac{1}{15} i \pi^{5} \zeta(3) \\
& \left.+\zeta(5) \zeta(3)+2 i \pi^{3} \zeta(5)-\frac{21}{2} i \pi \zeta(7)+\frac{\pi^{8}}{945}\right),
\end{aligned}
$$

which casts $\zeta(3,5)$ as the constant term of some linear combination of A-elliptic multiple zeta values. 


\subsection{Explicit formulae for A-elliptic multiple zeta values}

We now combine the results of the last two sections to write down explicit formulae for A-elliptic multiple zeta values in terms of iterated Eisenstein integrals and multiple zeta values. Denote by (cf. [6], eq. (4.2))

$$
\gamma\left(k_{1}, k_{2}, \ldots, k_{r} ; \tau\right)=(2 \pi i)^{-r} \mathscr{G}\left(k_{r}, \ldots, k_{2}, k_{1} ; \tau\right)
$$

the shuffle-regularized iterated integral of the Eisenstein series $G_{k_{1}}, \ldots, G_{k_{r}}$ as in Section 5.1 (the scaling factor $(-2 \pi i)^{-r}$ is adapted to the differential equation satisfied by A-elliptic multiple zeta values (74)).

\subsubsection{Length one}

Let us begin by completely giving all A-elliptic multiple zeta values of length one. Comparing coefficients on both sides of (74), one sees that

$$
2 \pi i \frac{\partial}{\partial \tau} I^{\mathrm{A}}(n ; \tau)=0
$$

Thus $I^{\mathrm{A}}(n ; \tau)$ is constant, and the precise value can be obtained from (78) as

$$
I^{\mathrm{A}}(n ; \tau)= \begin{cases}-2 \zeta(n) & n \text { even } \\ 0 & \text { else }\end{cases}
$$

in accordance with the results of Section 4.3.

\subsubsection{Length two}

We now move on to the length two case. In even weight, we already know the answer by the results of Section 4.3.2: it is

$$
I^{\mathrm{A}}\left(n_{1}, n_{2} ; \tau\right)= \begin{cases}0 & n_{1}, n_{2} \text { odd } \\ 2 \zeta\left(n_{1}\right) \zeta\left(n_{2}\right) & n_{1}, n_{2} \text { even } .\end{cases}
$$

Alternatively, this result could have also been obtained by a similar method as in the length one case, using the differential equation (74) and the constant term procedure (77). In the odd weight case, we encounter non-trivial iterated Eisenstein integrals for the first time: By (74), we see that for odd $n$

$$
2 \pi i \frac{\partial}{\partial \tau} I^{\mathrm{A}}(0, n ; \tau)=-2 n \zeta(n+1) G_{0}(\tau)-n G_{n+1}(\tau) .
$$

Together with (77), this gives, all in all 


$$
I^{\mathrm{A}}(0, n ; \tau)=\delta_{1, n} \frac{\pi i}{2}+n(\gamma(n+1 ; \tau)+2 \zeta(n+1) \gamma(0 ; \tau)) .
$$

The general case of an A-elliptic multiple zeta value of length two and odd weight can be reduced to this case, using the formula

$$
\begin{aligned}
I^{\mathrm{A}}\left(n_{1}, n_{2} ; \tau\right) & =(-1)^{n_{1}} I^{\mathrm{A}}\left(0, n_{1}+n_{2} ; \tau\right) \\
& +2 \delta_{n_{1}, 1} \zeta\left(n_{2}\right) I^{\mathrm{A}}(0,1 ; \tau)-2 \delta_{n_{2}, 1} \zeta\left(n_{1}\right) I^{\mathrm{A}}(0,1 ; \tau) \\
& +2 \sum_{p=1}^{\left\lceil\frac{1}{2}\left(n_{2}-3\right)\right\rceil}\left(\begin{array}{c}
n_{1}+n_{2}-2 p-2 \\
n_{1}-1
\end{array}\right) \zeta\left(n_{1}+n_{2}-2 p-1\right) I^{\mathrm{A}}(0,2 p+1 ; \tau) \\
& -2 \sum_{p=1}^{\left\lceil\frac{1}{2}\left(n_{1}-3\right)\right\rceil}\left(\begin{array}{c}
n_{1}+n_{2}-2 p-2 \\
n_{2}-1
\end{array}\right) \zeta\left(n_{1}+n_{2}-2 p-1\right) I^{\mathrm{A}}(0,2 p+1 ; \tau),
\end{aligned}
$$

(cf. [6], eq. (2.33)). This identifies A-elliptic multiple zeta values of length two as certain linear combinations of products of Eisenstein integrals and powers of $2 \pi i$.

\subsubsection{Length three}

We end this section by giving explicit formulae for A-elliptic multiple zeta values of length three. Given the simplicity of (87), it is natural to first compute $I^{\mathrm{A}}(0,0, n ; \tau)$ in terms of iterated integrals of Eisenstein series. Using again the differential equation together with the constant term procedure, one finds

$I^{\mathrm{A}}(0,0, n ; \tau)= \begin{cases}\delta_{1, n} \frac{\pi i}{4}+n\left(\frac{1}{2} \gamma(n+1 ; \tau)+\zeta(n+1) \gamma(0 ; \tau)\right) & n \text { odd } \\ -\frac{1}{3} \zeta(n)-n(n+1)(\gamma(n+2,0 ; \tau)+2 \zeta(n+2) \gamma(0,0 ; \tau)) & n \text { even }\end{cases}$

Using reflection and shuffle product formulae, the A-elliptic multiple zeta values $I^{\mathrm{A}}(0, n, 0 ; \tau)$ and $I^{\mathrm{A}}(n, 0,0 ; \tau)$ are readily expressed using $I^{\mathrm{A}}(0,0, n ; \tau)$ and products of A-elliptic multiple zeta values of lower length:

$$
\begin{aligned}
& I^{\mathrm{A}}(n, 0,0 ; \tau)=(-1)^{n} I^{\mathrm{A}}(0,0, n ; \tau), \\
& I^{\mathrm{A}}(0, n, 0 ; \tau)=I^{\mathrm{A}}(0, n ; \tau) I^{\mathrm{A}}(0 ; \tau)-I^{\mathrm{A}}(0,0, n ; \tau)-I^{\mathrm{A}}(n, 0,0 ; \tau) .
\end{aligned}
$$

Moreover, up to and including weight 7, every A-elliptic multiple zeta value of weight $n$ can be expressed as linear combinations of $I^{\mathrm{A}}(0,0, n ; \tau)$ and products, homogeneous of weight $n$, of powers of $2 \pi i$ and A-elliptic multiple zeta values of lower length (cf. [6], Section 2.2).

However, beginning with weight 8 , the situation changes. For example, 


$$
\begin{aligned}
I^{\mathrm{A}}(0,3,5 ; \tau)= & -405 \gamma(10,0 ; \tau)-75 \gamma(6,4 ; \tau)-\zeta(6)(150 \gamma(0,4 ; \tau)+90 \gamma(4,0 ; \tau)) \\
& -1008 \zeta(10) \gamma(0,0 ; \tau)
\end{aligned}
$$

and since $\gamma(6,4 ; \tau)$ appears in $(92)$, but neither in $(89)$, nor in any $\mathbb{Q}$-linear combination of A-elliptic multiple zeta values of lengths one or two, this shows that $I^{\mathrm{A}}(0,3,5 ; \tau)$ cannot be expressed using $I^{\mathrm{A}}(0,0,8 ; \tau)$ and lower length A-elliptic multiple zeta values alone.

\subsection{A special algebra of derivations}

It turns out that the precise linear combinations of iterated Eisenstein integrals, which appear in any linear combination of A-elliptic multiple zeta values are controlled by a special algebra of derivations $\mathfrak{u}^{\text {geom }}$ of the free Lie algebra $\mathbb{L}(x, y)$ over $\mathbb{Q}$ on two generators, which we describe next.

Consider the Lie algebra $\operatorname{Der}^{\Theta} \mathbb{L}(x, y)$ of derivations of $\mathbb{L}(x, y)$, which map the commutator $[x, y]$ to zero. There exist distinguished elements $\varepsilon_{2 k} \in \operatorname{Der}^{\Theta}(x, y)$, for $k \geq 0$, which satisfy

$$
\varepsilon_{2 k}(x)=\operatorname{ad}^{2 k}(x)(y),
$$

and their value on $y$ is uniquely determined by demanding that they be homogeneous for the bigrading $(*, *)$ on $\mathbb{L}(x, y)$, under which $x$ has bidegree $(1,0)$ and $y$ has bidegree $(0,1)$. Explicitly (cf. [37], eq. (2))

$$
\begin{aligned}
\varepsilon_{0}(y) & =0 \\
\varepsilon_{2 k}(y) & =\sum_{0 \leq j<k}(-1)^{j}\left[\operatorname{ad}^{j}(x)(y), \operatorname{ad}^{2 k-1-j}(x)(y)\right] .
\end{aligned}
$$

Definition 7. Define $\mathfrak{u}^{\text {geom }} \subset \operatorname{Der}^{\Theta} \mathbb{L}(x, y)$ to be the Lie subalgebra spanned by the $\varepsilon_{2 k}$.

The derivations $\varepsilon_{2 k}$ occur for example in $[2,16,32,37]$. They are related to universal mixed elliptic motives [28], as well as to polar solutions of the linearized double shuffle equations $[14,13]$.

By work of Pollack [37], the derivations $\varepsilon_{2 k}$ satisfy many relations, which are linked to the existence of cusp forms for $\mathrm{SL}_{2}(\mathbb{Z})$. In [6], Section 4.3, it is described how relations between $\varepsilon_{2 k}$ constrain the linear combinations of iterated Eisenstein integrals which can possibly appear as A-elliptic multiple zeta values. The starting point is the generating series of A-elliptic multiple zeta values

$$
e^{-\pi i[x, y]} A(\tau)=\widetilde{A}(\tau)=\sum_{r \geq 0}(-1)^{r} \sum_{n_{1}, \ldots, n_{r} \geq 0} I^{\mathrm{A}}\left(n_{1}, \ldots, n_{r} ; \tau\right) \operatorname{ad}^{n_{r}}(x)(y) \ldots \mathrm{ad}^{n_{1}}(x)(y) .
$$

By [22], eq. (7), and since every $\varepsilon_{2 k}$ annihilates $[x, y]$, this series satisfies the differential equation 


$$
\left.2 \pi i \frac{\partial}{\partial \tau} \widetilde{A}(\tau)=-\sum_{k \geq 0}(2 k-1) G_{2 k}(\tau) \varepsilon_{2 k}(\widetilde{A}(\tau))\right),
$$

which is in fact equivalent to Theorem 5, as shown in [22], Section 4. Note that in (97), the derivation $\varepsilon_{2 k}$ is coupled to the Eisenstein series $G_{2 k}(\tau)$. Iteratively integrating this differential equation leads to a coupling of iterated integrals of Eisenstein series of length $r$ and commutators of derivations $\varepsilon_{2 k}$ of depth $r$.

As a simple example, consider the "Ihara-Takao relation" in $\mathfrak{u}^{\text {geom }}$

$$
\left[\varepsilon_{10}, \varepsilon_{4}\right]-3\left[\varepsilon_{8}, \varepsilon_{6}\right]=0
$$

whose existence can be traced back to the unique, up to a scalar, cusp form for $\mathrm{SL}_{2}(\mathbb{Z})$ of weight 12 [37]. It implies that the iterated Eisenstein integrals $\gamma(10,4 ; \tau)$ and $\gamma(8,6 ; \tau)$ only appear in a special ratio. More precisely, it follows from the differential equation (97) and from (98) that the only linear combination of the two double Eisenstein integrals, which appears as an A-elliptic multiple zeta value is, up to a scalar, given by

$$
81 \gamma(10,4 ; \tau)+35 \gamma(8,6 ; \tau) \text {. }
$$

If one introduces a different normalization of iterated Eisenstein integrals, namely

$$
\underline{\gamma}\left(k_{1}, \ldots, k_{r} ; \tau\right)=\prod_{i=1}^{r}\left(k_{i}-1\right) \gamma\left(k_{1}, \ldots, k_{r} ; \tau\right)
$$

then (99) becomes

$$
3 \underline{\gamma}(10,4 ; \tau)+\underline{\gamma}(8,6 ; \tau)
$$

Viewing $\underline{\gamma}\left(k_{1}, k_{2} ; \tau\right)$ as dual to $\left[\varepsilon_{k_{1}}, \varepsilon_{k_{2}}\right]$, we see that (101) is orthogonal to the relation (98). In this way, the linear combinations of iterated Eisenstein integrals which appear in elliptic multiple zeta values are orthogonal to relations between commutators of the $\varepsilon_{2 k}$ (see also [12], Section 12). For much more detailed treatments of the relation between $\mathfrak{u}^{\text {geom }}$ and elliptic multiple zeta values, see $[6,33,35]$.

Acknowledgements Many thanks to the organizers of the Research Trimester on Multiple Zeta Values, held September-December 2014 at ICMAT, Madrid, where part of this research was carried out. This paper contains results obtained in joint work with Johannes Broedel, Carlos Mafra and Oliver Schlotterer, and I would like to thank them very much. Incidentally, that collaboration started after the author gave a talk at the ICMAT in September 2014, as part of this research trimester. Also, many thanks to Henrik Bachmann, Johannes Broedel, Ulf Kühn and Oliver Schlotterer for helpful comments, as well as the Albert-Einstein-Institute in Potsdam, the Department of Applied Mathematics and Theoretical Physics in Cambridge and the Mainz Institute for Theoretical Physics for hospitality. This work is part of the author's PhD thesis at Universität Hamburg, and I would like to thank my advisor Ulf Kühn for his constant support of my work and for his encouragement. 


\section{References}

1. Bannai, K., Kobayashi, S., Tsuji, T.: On the de Rham and p-adic realizations of the elliptic polylogarithm for CM elliptic curves. Ann. Sci. École. Norm. Sup. (4), 43(2):185-234, 2010.

2. Baumard, S., Schneps, L.: On the derivation representation of the fundamental Lie algebra of mixed elliptic motives. Ann. Math. Qué., 41(1):43-62, 2017.

3. Bloch, S. J.: Higher regulators, algebraic K-theory, and zeta functions of elliptic curves, volume 11 of CRM Monograph Series. American Mathematical Society, Providence, RI, 2000.

4. Broadhurst, D. J., Kreimer, D.: Association of multiple zeta values with positive knots via Feynman diagrams up to 9 loops. Phys. Lett. B, 393(3-4):403-412, 1997.

5. Broedel, J., Mafra, C. R., Matthes, N., Schlotterer, O.: Elliptic multiple zeta values and oneloop superstring amplitudes. J. High Energy Phys. 2015, no. 7, 112, front matter+41 pp.

6. Broedel, J., Matthes, N., Schlotterer, O.: Relations between elliptic multiple zeta values and a special derivation algebra. J. Phys. A 49 (2016), no. 15, 155203, 49 pp.

7. Broedel, J., Schlotterer, O., Stieberger, S.: Polylogarithms, multiple zeta values and superstring amplitudes. Fortschr. Phys., 61(9):812-870, 2013.

8. Broedel, J., Schlotterer, O., Stieberger, S., Terasoma T.: All order $\alpha^{\prime}$-expansion of superstring trees from the Drinfeld associator. Phys. Rev., D89(6):066014, 2014.

9. Brown, F.: Mixed Tate motives over $\mathbb{Z}$. Ann. of Math. (2) 175 (2012), no. 2, 949-976.

10. Brown, F.: Iterated integrals in quantum field theory. Geometric and topological methods for quantum field theory, 188-240, Cambridge Univ. Press, Cambridge, 2013.

11. Brown, F.: Depth-graded motivic multiple zeta values. arXiv:1301.3053.

12. Brown, F.: Multiple modular values and the relative completion of the fundamental group of $\mathscr{M}_{1,1}$. arXiv:1407.5167v3.

13. Brown, F.: Zeta elements in depth 3 and the fundamental Lie algebra of the infinitesimal Tate curve. Forum Math. Sigma, 5:e1, 56, 2017.

14. Brown, F.: Anatomy of an associator. arXiv:1709.02765.

15. Brown, F., Levin, A.: Multiple elliptic polylogarithms. arXiv:1110.6917.

16. Calaque, D., Enriquez, B., Etingof, P.: Universal KZB equations: the elliptic case. In Algebra, arithmetic, and geometry: in honor of Yu. I. Manin. Vol. I, volume 269 of Progr. Math., pages 165-266. Birkhäuser Boston, Inc., Boston, MA, 2009.

17. Chen, K. T.: Iterated path integrals. Bull. Amer. Math. Soc., 83(5):831-879, 1977.

18. Deligne, P.: Le groupe fondamental de la droite projective moins trois points. In Galois groups over $\mathbb{Q}$ (Berkeley, CA, 1987), volume 16 of Math. Sci. Res. Inst. Publ., pages 79297. Springer, New York, 1989.

19. Deligne, P., Goncharov, A. B.: Groupes fondamentaux motiviques de Tate mixte. Ann. Sci. École Norm. Sup. (4) 38 (2005), no. 1, 1-56.

20. Drinfel'd, V. G.: On quasitriangular quasi-Hopf algebras and on a group that is closely connected with $\operatorname{Gal}(\overline{\mathbb{Q}} / \mathbb{Q})$. Leningrad Math. J. 2 (1991), no. 4, 829-860.

21. Enriquez, B.: Elliptic associators. Selecta Math. (N.S.) 20 (2014), no. 2, 491-584.

22. Enriquez, B.: Analogues elliptiques des nombres multiztas. Bull. Soc. Math. France 144 (2016), no. 3, 395-427.

23. Furusho, H.: Double shuffle relation for associators. Ann. of Math. (2), 174(1):341-360, 2011.

24. Gangl, H., Kaneko, M., Zagier, D.: Double zeta values and modular forms. In Automorphic forms and zeta functions, pages 71-106. World Sci. Publ., Hackensack, NJ, 2006.

25. Goncharov, A. B.: Multiple polylogarithms, cyclotomy and modular complexes. Math. Res. Lett., 5(4):497-516, 1998.

26. Goncharov, A. B., Manin, Y. I.: Multiple $\zeta$-motives and moduli spaces $\mathscr{M}_{0, n}$. Compos. Math. 140 (2004), no. 1, 1-14.

27. Hain, R. M.: The geometry of the mixed Hodge structure on the fundamental group. In $\mathrm{Al}$ gebraic geometry, Bowdoin, 1985 (Brunswick, Maine, 1985), volume 46 of Proc. Sympos. Pure Math., pages 247-282. Amer. Math. Soc., Providence, RI, 1987.

28. Hain, R., Matsumoto M.: Universal Mixed Elliptic Motives. arXiv:1512.03975. 
29. Knizhnik, V. G., Zamolodchikov, A. B.: Current algebra and Wess-Zumino model in two dimensions. Nuclear Phys. B 247 (1984), no. 1, 83-103.

30. Le, T. T. Q., Murakami, J.: Kontsevich's integral for the Kauffman polynomial. Nagoya Math. J. 142 (1996), 39-65.

31. Levin, A.: Elliptic polylogarithms: an analytic theory. Compositio Math., 106(3):267-282, 1997.

32. Levin, A., Racinet, G.: Towards multiple elliptic polylogarithms. arXiv:math/0703237.

33. Lochak, P., Matthes, N., Schneps, L.: Elliptic multizetas and the elliptic double shuffle relations, arXiv: 1703.09410.

34. Manin, Y. I.: Iterated integrals of modular forms and noncommutative modular symbols. In Algebraic geometry and number theory, volume 253 of Progr. Math., pages 565-597. Birkhäuser Boston, Boston, MA, 2006.

35. Matthes, N.: Elliptic multiple zeta values. PhD thesis, Universität Hamburg, 2016.

36. Matthes, N.: Elliptic double zeta values. J. Number Theory 171 (2017), 227-251.

37. Pollack, A.: Relations between derivations arising from modular forms. Master's thesis, Duke University, 2009.

38. Racinet, G.: Doubles mélanges des polylogarithmes multiples aux racines de l'unité. Publ. Math. Inst. Hautes Études Sci., (95):185-231, 2002.

39. Ree, R.: Lie elements and an algebra associated with shuffles. Ann. of Math. (2), 68:210$2220,1958$.

40. Schlotterer, O., Stieberger, S.: Motivic multiple zeta values and superstring amplitudes. J. Phys. A, 46(47):475401, 37, 2013.

41. Terasoma, T.: Geometry of multiple zeta values. In International Congress of Mathematicians. Vol. II, pages 627?635. Eur. Math. Soc., Zürich, 2006.

42. Weil, A.: Elliptic functions according to Eisenstein and Kronecker. Springer-Verlag, BerlinNew York, 1976. Ergebnisse der Mathematik und ihrer Grenzgebiete, Band 88.

43. Zagier, D.: The Bloch-Wigner-Ramakrishnan polylogarithm function. Math. Ann., 286(13):613-624, 1990

44. Zagier, D.: Periods of modular forms and Jacobi theta functions. Invent. Math., 104(3):449465, 1991.

45. Zagier, D.: Values of zeta functions and their applications. In First European Congress of Mathematics, Vol. II (Paris, 1992), volume 120 of Progr. Math., pages 497-512. Birkhäuser, Basel, 1994. 